\title{
Alcohol and Canadian Health
}

\author{
James McIntosh $^{1}$ \\ ${ }^{1}$ Economics Department, Concordia University, Quebec, Canada \\ Correspondence: James McIntosh, Economics Department, Concordia University, 1455 De Maisonneuve Blvd. W. \\ Montreal Quebec, H3G 1M8, Canada and 623 Econometrics, 59 Rue Saint Viateur E. Montreal, Quebec, EH2T \\ 1A7, Canada. E-mail: james.mcintosh@concordia.ca
}

\author{
Received: January 10, 2017 Accepted: February 14, 2017 Online Published: February 28, 2017 \\ doi:10.5539/gjhs.v9n5p96 URL: https://doi.org/10.5539/gjhs.v9n5p96
}

\begin{abstract}
Background: The role of alcohol use as a cause of disease and a determinant of individual health has been examined by a large number of researchers and is an important policy issue.

Methods: The effect of alcohol use on the four most important diseases that afflict Canadians is examined by applying finite mixture probability models to data from the 2011-12 Canadian Community Health Survey. The effect on self-reported health is also considered.

Results: Regular drinking behaviour is shown to be associated with lower probabilities of having diabetes, coronary heart disease, or stroke, although it may lead to a higher probability of having cancer for some sub-populations. The net prophylactic effect of moderate alcohol use on diseases diseases other than cancer is positive and significant. Men and women in all age groups report higher health status scores as a result of regular alcohol use. Regular drinking also leads to fewer doctor visits.
\end{abstract}

Conclusions: The fears and apprehension expressed by so many health researchers ignore the substantial beneficial effects that regular drinkers experience. Alcohol policy should therefore be less repressive more sympathetic to consumer wants.

Keywords: alcohol, self-perceived health, cancer, coronary heart disease, diabetes, stroke, Canada

\section{Introduction}

Much has been written about the health problems that arise from the recreational consumption of alcohol. Often the focus is on alcohol abuse but there are a number of researchers who express concerns over moderate alcohol use as well. Room et al (2005), Boffeta and Hashibe (2006), Rhem et al (2006), Li (2008), Lauer and Sorlie (2009) all stress the negative effects of alcohol use. But there is an extensive literature on the effects of alcohol on mortality and health which takes a more favorable view. The main findings in this literature are summarized by O'Keefe et al. (2007: 1009) by saying

"An extensive body of data shows concordant J-shaped associations between alcohol intake and a variety of adverse health outcomes, including coronary heart disease, diabetes, hypertension, congestive heart failure, stroke, dementia, Raynaud's phenomenon, and all-cause mortality. Light to moderate alcohol consumption (up to 1 drink daily for women and 1 or 2 drinks daily for men) is associated with cardioprotective benefits, whereas increasingly excessive consumption results in proportional worsening of outcomes".

Most of the studies to which they refer and more recent ones are longitudinal in nature and explain either how life durations or times to disease onset relate to alcohol consumption. Newer contributions to the all-cause and competing risk mortality literature include Fuchs et al. (1995), Di Castelnuovo et al. (2006), Klatsky and Udaltsova (2007), Baer et al. (2010), Fuller et al (2011) and Bergmann et al (2013). All of these studies support what O'keefe claims. For coronary heart disease, diabetes, and ischemic stroke there are recent meta-studies which also confirm this J-shaped relation; these are Roerecke et al. (2010, 2012: Table 2), Baliunas et al. (2009: Figure 3), and Patra et al. (2010: Figure 7), respectively, for diseases other than cancer.

With the exception of cancer, the results reported here are similar to what others have found using prospective cohort data. However, what is different in this study is the type of data employed. This study uses two cross-section surveys for which there is only one observation at one point in time for each respondent. It will be argued in section 3 that this is a legitimate procedure since alcohol use is more or less constant over large age ranges and the 
statistical procedure employed (mixture models) can deal with the small number of respondents whose present alcohol consumption behavior differs from their historical behavior.

This study examines the effects of alcohol consumption on the probability that an individual will have cancer, coronary heart disease, diabetes and stroke, four leading causes of Canadian ill-health. It also examines the effect of drinking behavior on self-reported health.

It has the following structure. The first section deals with the effects of alcohol on cause specific mortality and why these results are often misinterpreted. Section 2 analyzes the effect of alcohol consumption on self-perceived health using data from two Canadian Community Health Surveys, Statistics Canada (2009, 2013). Results are discussed in the next section and the paper ends with a discussion of the results and some conclusions.

\section{Data and Variables}

The data used in this study comes from a series of Canadian Community Health Surveys with the main focus on the 2011-12 survey. Statistics Canada carries out large detailed surveys on the health of Canadians on a regular basis. The focus of attention is on a recent pair of surveys carried out in 2011 and 2012 involving more than 130000 representative Canadians although for comparison purposes some reference is made to the 2007-08 surveys. Statistics Canada collects information on health outcomes, drinking, smoking, dietary, and exercise behaviour as well a large number of individual socio-demographic characteristics.

In addition to the variables listed in Table 1 information on the respondent's educational attainment - 5 categories, smoking behaviour - 7 categories, marital status - 2 categories, geographic region - 5 categories, body mass index, and income, were used in the statistical analysis. Alcohol use is represented by a frequency variable. There are eight frequencies. These are listed in Table 3. The representation of alcohol use was preferred to the number of drinks consumed per day or per week since it is less vulnerable to recall error. Data on drinks per day or week also does not agree with published data on per-capita alcohol sales.

Table 1. Summary Statistics for Selected Variables Males And Females, 2011-2012

\begin{tabular}{lll}
\hline Frequency Of Drinking & Males & Females \\
\hline Non-Drinker & 0.200 & 0.275 \\
Less Than Once A Month & 0.115 & 0.207 \\
Once A Month & 0.065 & 0.080 \\
$\mathbf{2}$ To 3 Times A Month & 0.085 & 0.091 \\
Once A Week & 0.130 & 0.109 \\
$\mathbf{2}$ To 3 Times A Week & 0.192 & 0.131 \\
$\mathbf{4}$ To 6 Times A Week & 0.077 & 0.040 \\
Every Day & 0.134 & 0.066 \\
\hline Diseases & & \\
Cancer & 0.036 & 0.042 \\
Coronary Heart Disease & 0.106 & 0.076 \\
Diabetes & 0.119 & 0.093 \\
Stroke & 0.022 & 0.019 \\
\hline Self-Reported Health & & \\
Poor & 0.047 & 0.041 \\
Fair & 0.120 & 0.119 \\
Good & 0.321 & 0.301 \\
Very Good & 0.350 & 0.364 \\
Excellent & 0.163 & 0.175 \\
Sample Size & 42185 & 54060 \\
\hline & & \\
\hline
\end{tabular}


The term 'regular drinker' is used from time to time in the text. A regular drinker consumes a standard drink (12.5 $\mathrm{gm}$ of alcohol) at least once a week. Statistics Canada has a different definition. It defines a regular drinker as one who consumes alcohol at least once a month. This is not a good definition since there are significant differences across the sub-categories within the category at least once a month. This is also true for the term itself so it is never used in a statistical model as a category. There is also a variable called 'heavy drinker'. This is defined here as drinking 5 or more drinks on one occasion at least once a week.

All models use normalized regressors. These are variables which have been transformed to zero mean unit variance variables. When these are used the size of the regression coefficient represents the importance of the variable. Inferences about significance are not affected by the transformation.

All individuals who reported their health status, were age 30 or over, and did not reside is any of the territories are included in the analysis. The restricted sample contains 42185 males and 54060 females. Respondents under age 30 do not usually suffer from the diseases considered here and were excluded for that reason. The analysis was gender specific and all models were run on three age groups: 30-49, 50-69 and 70 +. Ten year age groups were considered but the additional age disaggregation added nothing to the analysis. Statistics Canada provided sample weights which make the survey representative of the entire population. It recommends their use in any type of regression procedure. They were used here, although for most parameters there was very little difference between weighted and un-weighted estimates.

\section{Methods and Statistical procedures}

Let $\left\{y_{i j}=1\right\}$ be the event that respondent $i$ has disease $j$ and let the probability of this event be

$$
\operatorname{Pr}\left\{y_{i j}=1\right\}=F_{j}\left(X_{i}\right)
$$

where $\mathrm{X}_{\mathrm{i}}$ is a vector of characteristics of respondent $\mathrm{i}$ and $\mathrm{F}$ is a cumulative distribution function. Some of the elements in $X_{i}$ represent alcohol use behaviour at the time of the survey. Using data like this to explain whether alcohol consumption has any effect on whether a person has a particular disease is often seen as problematic since disease probabilities are usually associated with life-time and not current alcohol use. This explains the preference for longitudinal data for this type of analysis. However, has been recently shown by McIntosh (2014), Canadian alcohol consumption behaviour is very stable over large age ranges for most adults and begins to decline significantly only after age 70 . These results are confirmed by the data in Table 2 .

Table 2. Proportion of Regular Drinkers In Canadian Community Health Surveys: 2007-08 and 2011-12

\begin{tabular}{|c|c|c|c|c|}
\hline \multirow{2}{*}{$\begin{array}{l}\text { Genders } \\
\text { Age Group }\end{array}$} & \multicolumn{2}{|l|}{ Males } & \multicolumn{2}{|c|}{ Females } \\
\hline & $2007-8$ & 2011-12 & $2007-8$ & 2011-12 \\
\hline $20-29$ & 0.553 & 0.554 & 0.340 & 0.355 \\
\hline 30-39 & 0.552 & 0.549 & 0.324 & 0.356 \\
\hline $40-49$ & 0.549 & 0.544 & 0.393 & 0.391 \\
\hline 50-59 & 0.572 & 0.524 & 0.404 & 0.416 \\
\hline 60-69 & 0.537 & 0.549 & 0.354 & 0.360 \\
\hline 70-79 & 0.476 & 0.480 & 0.259 & 0.271 \\
\hline $80+$ & 0.372 & 0.383 & 0.184 & 0.200 \\
\hline Average & 0.533 & 0.536 & 0.335 & 0.347 \\
\hline \multicolumn{5}{|l|}{ Sample } \\
\hline Size & 24807 & 49058 & 30872 & 62118 \\
\hline
\end{tabular}

Since this is not true for the entire sample a method which deals with respondents whose current alcohol consumption behaviour differs from life-time behaviour is needed.

The procedure to be used here uses latent class analysis. Suppose there is a typology of respondents where type A respondents have not changed their drinking habits since being an adolescent and type $\mathrm{B}$ have. Let the two disease probability distributions for disease $\mathrm{j}$ be $\mathrm{F}_{\mathrm{jA}}$ and $\mathrm{F}_{\mathrm{jB}}$ and let the probability of being type $A$ and $\mathrm{B}$ be $\mathrm{P}_{\mathrm{jA}}$ and $\mathrm{P}_{\mathrm{jB}}$, respectively. The probability distribution for the latent class model is the mixture distribution 


$$
F_{j}\left(X_{i}\right)=P_{j A} F_{j A}\left(X_{i}\right)+P_{j B} F_{j}\left(X_{i}\right)
$$

In (2) the presence of the two latent classes allows the probability of having disease $\mathrm{j}$ to depend on the individual's type. This is done here by allowing the mean of each distribution to be a linear function of the regressors associated with drinking behaviour which is specific to each type. Formally, this means that

$$
\mu_{j A}=X_{i} \alpha \text { and } \mu_{j B}=X_{i} \beta
$$

Where the parameters are the same for all respondents and $\alpha=\beta$ for all non-alcohol related regressors.

\section{Results}

Parameter estimates based on un-mixed models appear in Table 3 for males and females for the three age groups defined above.

Mixture models were estimated for each of the four diseases but these did not lead to an improvement in model performance. Unmixed models were always supported by the Bayesian information criterion as the best choice of model to explain the data.

For coronary heart disease, diabetes and stroke the probability of having the disease is lowest for the drinking categories of more than once a week. For coronary heart disease it is lowest for respondents who drink every day. Drinking 2 to 3 or 4 to 6 times a week also have large and significant negative regression coefficients associated with them. Regular alcohol use is most important for the age group 50-69 although it is also important for the other two age groups. Although, there are some differences across the two genders the results are broadly similar.

The results for cancer are quite different. For the age group 30-49 most of the drinking categories are associated with a significantly lower probability of having cancer. The regression coefficients are quite large especially for those male respondents who drink 4 to 6 times a week. For the other two age groups alcohol use has little effect and only 3 of the 28 cancer coefficients are significant. This result differs from what other researchers have found and will be discussed in the next section.

The results for self-reported health are based on an unmixed ordered probability model and they are similar to those for coronary heart disease, diabetes and stroke. In all of the models other regressors were included. Respondents who were well educated, married, not overweight, did not smoke, and for self-reported health, earning large incomes and were not heavy drinkers, were less likely to have any of the diseases considered here and had better health.

While the actual impact of drinking behaviour on health outcomes is not clear from Table 3, this can be determined from Table 4 which shows diseased prevalence rates by drinker category for the age group 50-69 which is most affected by alcohol use.

\begin{tabular}{|c|c|c|c|c|c|c|c|c|}
\hline $\begin{array}{l}\text { Males and } \\
\text { Females 2011-12 }\end{array}$ & Frequen & of Alcohol & onsumptio & & & & & \\
\hline \multicolumn{9}{|l|}{ Males } \\
\hline Age & & & & & & & & Heavy \\
\hline Group & 1 & 2 & 3 & 4 & 5 & 6 & 7 & Drinker \\
\hline \multicolumn{9}{|l|}{$30-49$} \\
\hline Cancer & -0.085 & $-0.196 * *$ & $-0.184 * *$ & $-0.200 *$ & $-0.248 * *$ & $-0.303 * *$ & -0.067 & -0.060 \\
\hline CHD & -0.016 & -0.002 & -0.077 & -0.051 & -0.061 & -0.044 & $-0.098 *$ & -0.029 \\
\hline Diabetes & 0.021 & $-0.088 * *$ & $-0.113 * *$ & $-0.147 * *$ & $-0.216^{* *}$ & $-0.172 * *$ & $-0.115^{*}$ & 0.013 \\
\hline Stroke & -0.030 & -0.159 & -0.003 & $-0.225 * *$ & $-0.211 * *$ & -0.210 & -0.005 & -0.030 \\
\hline SR-Health & -0.005 & 0.009 & $0.043 * *$ & $0.071 * *$ & $0.085 * *$ & $0.054 * *$ & $0.053 * *$ & $-0.034 *$ \\
\hline \multicolumn{9}{|l|}{$50-69$} \\
\hline Cancer & $0.061 * *$ & 0.007 & 0.032 & 0.028 & -0.026 & 0.052 & 0.014 & -0.009 \\
\hline CHD & -0.001 & -0.018 & $-0.056^{* *}$ & $-0.072 * *$ & $-0.099 * *$ & -0.026 & $-0.092 * *$ & 0.001 \\
\hline
\end{tabular}

Table 3. Weighted normalized parameter estimates for the effects of alcohol consumption 


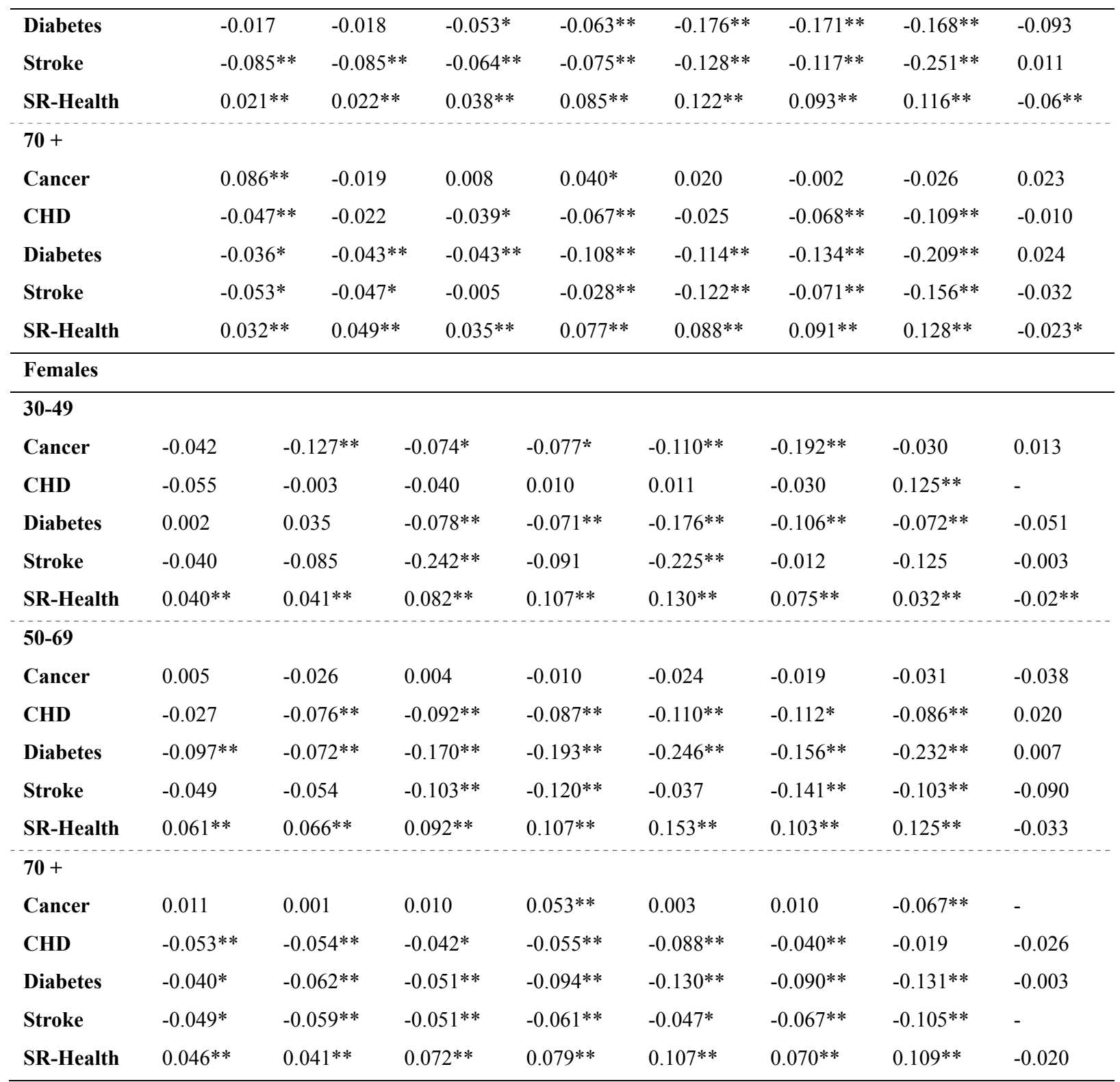

Notes. Frequencies start with 1, equal to less than once a month to 7, equal to every day. SR-Health is self- reported health. $*$ is significant at $5 \%$ and $* *$ significant at $1 \%$, respectively. 
Table 4. Proportions aged 50-69 with various diseases and excellent or very good health by frequency of alcohol use

\begin{tabular}{|c|c|c|c|c|c|c|c|}
\hline \multicolumn{8}{|c|}{ Males and Females 2011-2012 } \\
\hline \multicolumn{8}{|l|}{ Males } \\
\hline Frequency of Drinking & Cancer & CHD & Diabetes & Stroke & Very Good or & Excellent & Health \\
\hline Non -Drinker & 0.040 & 0.148 & 0.198 & 0.035 & 0.386 & & \\
\hline Less Than $1 \mathrm{~A}$ Month & 0.041 & 0.144 & 0.198 & 0.020 & 0.406 & & \\
\hline Once A Month & 0.033 & 0.119 & 0.173 & 0.023 & 0.433 & & \\
\hline 2 To 3 Times A Month & 0.042 & 0.093 & 0.145 & 0.023 & 0.467 & & \\
\hline Once A Week & 0.035 & 0.094 & 0.129 & 0.019 & 0.522 & & \\
\hline 2 To 3 Times A Week & 0.027 & 0.080 & 0.098 & 0.014 & 0.574 & & \\
\hline 4 To 6 Times A Week & 0.032 & 0.100 & 0.081 & 0.011 & 0.574 & & \\
\hline Every Day & 0.035 & 0.101 & 0.093 & 0.012 & 0.542 & & \\
\hline \multicolumn{8}{|l|}{ Females } \\
\hline Non -Drinker & 0.050 & 0.090 & 0.169 & 0.024 & 0.399 & & \\
\hline Less Than 1 A Month & 0.046 & 0.066 & 0.138 & 0.017 & 0.467 & & \\
\hline Once A Month & 0.040 & 0.046 & 0.115 & 0.014 & 0.533 & & \\
\hline 2 To 3 Times A Month & 0.052 & 0.045 & 0.062 & 0.008 & 0.593 & & \\
\hline Once A Week & 0.041 & 0.041 & 0.055 & 0.008 & 0.607 & & \\
\hline 2 To 3 Times A Week & 0.045 & 0.036 & 0.040 & 0.010 & 0.662 & & \\
\hline 4 To 6 Times A Week & 0.043 & 0.033 & 0.039 & 0.005 & 0.711 & & \\
\hline Every Day & 0.047 & 0.047 & 0.035 & 0.011 & 0.675 & & \\
\hline
\end{tabular}

The effect of regularly drinking 4 to 6 days per week has a huge impact on disease prevalence. Consider diabetes; $19.8 \%$ of non-drinking males had diabetes but only $8.1 \%$ of those males who drank 4 to 6 times a week had diabetes. The results are even larger for females, $16.9 \%$ compared to $3.9 \%$. Self-reported health displays the same pattern. Non-drinkers have much poorer health than respondents who drink more than 3 times a week. All of these rate differences are highly significant since the standard deviations of all of the rates in the Table 4 are less than 0.015 .

\section{Discussion}

The most controversial result here is the negative association between cancer and higher levels of alcohol consumption. There is a great deal of research which shows that alcohol is a cause of cancer. A recent survey of this literature Baan et al (2008) notes that "Many studies of different design and in different populations around the world have consistently shown that regular alcohol consumption is associated with an increased risk for cancers of the oral cavity, pharynx, larynx, and oesophagus". But other studies show that the major portion of the alcohol attributable burden of the incidence of cancer is associated with drinkers who exceed the recommended number of drinks per day. This may explain the lack of association between cancer and alcohol use for the two oldest age groups since there are few drinkers in these age groups who exceed drinking guidelines. Furthermore, some of the recent longitudinal all-cause mortality or competing risk studies find no association between alcohol use and the longevity of those who have cancer so the absence of a statistical relationship has some plausibility. However, there is no evidence in this literature that supports the proposition that moderate alcohol use acts as a prophylactic for cancer so the results reported here are quite unusual.

Many studies which examine the effects of alcohol consumption on health do this by considering how drinking behaviour affects life durations using longitudinal data. They assess respondent characteristics at baseline and then track the respondents over time to see whether their life durations are related to their alcohol consumption behaviour at baseline, or in some cases historical drinking behaviour. This procedure has statistical advantages since alcohol consumption behaviour is measured prior to the occurrence of the events of interest. Morbidity has also been examined in this way except durations are from baseline to the first occurrence of a disease. A review of 
the literature which discusses the benefits of using self-reported health data is contained in McMinn et al (2011).

But as argued in the methods section there is no advantage to be gained from this methodology if alcohol consumption behaviour is constant over the life course. If this is the case then information on drinking behaviour can be collected at any age. However, there are serious limitations with studies which are based on longitudinal data. First, all-cause mortality and competing risk studies focus on end of life events. To be sure, these are important; everyone wants to live longer but respondents are old when these events occur so this methodology tells us nothing about the costs or benefits of alcohol consumption for younger individuals. Hardly anyone dies between the ages of 30 and 49 so it is important to have welfare measures which are relevant to younger age groups. An individual's welfare very much depends on his or her health status and whether or not the person is free of these diseases. Self-reported health is a very good measure of individual welfare at whatever age it is collected and this makes it an attractive metric for the analysis of the effects of alcohol consumption on individuals.

Secondly, most studies delete respondents who are not disease free at baseline. When the sample populations have older respondents excluding those who suffer from coronary heart disease or diabetes will produce a biased sample since the proportions in the age group 50-69 who have these two diseases is quite large. Sample selection problems can be quite serious and it has been shown by Bergmann et al (2013) that they may contaminate the results.

It is clear that there are different types of survey that can be informative on this issue. They should be viewed as being complementary and not competing alternatives. The use of sample cross-section surveys can add to the results that are obtained from studies based on longitudinal data. The increase in lifetimes of regular drinkers who die of coronary heart disease is partly explained by the data in Table 4 which shows that respondents who do suffer from coronary heart disease are will have experienced its onset later if they have been drinking alcoholic beverages regularly.

In the Canadian case there is no alternative to the cross section data in the Canadian Community Health Surveys since neither Statistics Canada nor any other Canadian organization has organized a suitable longitudinal health survey. The one Canadian survey that does exist, the National Population Health Survey, Statics Canada (2013) contains no information on age at or cause of death and there is considerable attrition in the survey $(31.3 \%$ from 1994-95 to 2010-11). As a result there is no choice and all that can be done is to make the best use of the data that is available.

In issues like alcohol use and health there is question of causality. Does moderate alcohol use lead to better health outcomes or do people drink moderately because they feel good? The results here favour the first alternative. First, as is shown in Table 2 drinking behaviour is relatively stable over age groups under 70 and this is not true of self-reported health or any of the diseases considered here so that current health status cannot explain current drinking behaviour. The relations between diseases also appear to run from moderate alcohol use to better health outcomes rather than the other way around. Some studies mention physiological mechanisms whereby moderate alcohol use directly promotes better health. O'keefe et al (2007: 1010) note that 'alcohol, when used in moderation, has an anti-atheriosclerotic effect ... and confers cardiovascular protection predominantly through enhancement of insulin sensitivity and elevation of high density lipoprotein cholesterol'. It is also the case that medical advice does not usually recommend diminished alcohol use or abstinence as part of the treatment for any of the diseases involved here so the case for moderate alcohol use causing better health outcomes is quite compelling. Research on doctor visits suggests the same conclusion, McIntosh (2014). For the relation between doctor visits and moderate alcohol use it is clear that moderate alcohol use reduces these and not the other way around.

There is one final issue which needs to be addressed and that involves the higher rates of disease among non-drinkers. It has been alleged by some researchers, Fillmore et al (2006) among others, that this result is a consequence of a classification error which arises when former drinkers are included in the non-drinker category. The health of former drinkers is usually found to be more problematic than that of non-drinkers and it is their presence in the non-drinking category that makes the regular drinkers appear to be more healthy or less subject to the diseases discussed here. This problem was investigated in McIntosh (2009) which showed that although the classification error makes a difference in the results the effect is not large enough to invalidate the conclusions of this study.

\section{Conclusions}

As noted earlier, the message from some scholars in the alcohol research community is that individuals should drink less and therefore avoid the wide variety of diseases that are associated with alcohol consumption. A good example of this is Rhem et al. (2009: 2273) who state that the net effect of alcohol consumption on health is detrimental'. This misses the essential point that individuals get utility from drinking and the quality of their health 
is significantly better when they drink moderately. Moderate alcohol use also reduces all-cause mortality, disease prevalence, and the degree to which respondents use the health service. Alcohol is like any other consumption good. People spend money on it because it gives them pleasure and they feel better health-wise because of it. In contemporary economic theory measures of social welfare are based on individual preferences. In a utilitarian framework social welfare is the sum of individual welfares. In Canada the vast majority $(81.1 \%$ of men and $74.0 \%$ of women) of Canadians as regular or occasional drinkers benefit from drinking moderately (Note 1). If Canada has a problem it is under consuming alcohol not drinking excessively. Heavy episodic drinking is rare except for males aged 20-29 and average consumption according to Statistics Canada is 8 litres per year (1.27 drinks per day for the population over age 15). Even if men drink twice as much as women the average number of drinks per day is well below what the safe drinking guidelines outlined in Stockwell (2012). If more Canadians drank moderately this would increase aggregate welfare not reduce it.

To be sure there are individuals who are unable to use alcohol responsively and this leads to serious externalities in terms of increased traffic accidents, anti-social behaviour, and the high costs of alcoholism both to families and the health system. However, restrictive policies in terms of high consumption taxes and limits to availability by making it inconvenient to buy alcoholic beverages punish the majority for the sins of the few. Consumers are worse off because of them.

\section{Competing Interests Statement}

The author declares that there is no conflict of interests regarding the publication of this paper.

\section{References}

Baan, R., Straif, K., Grosse, Y., Secretan, B., El Ghissassi, F., Bouvard, V., ... Cogliano, V. (2007). Carcinogenicity of alcoholic beverages. Lancet Oncology, 8(4), 292-293. https://doi.org/10.1016/S1470-2045(07)70099-2

Baer, H. J., Glynn, R. J., Hu, F. B., Hankinson, S. E., Willett, W. C., Colditz, G. A., ... \& Rosner, B. (2011). Risk factors for mortality in the nurses' health study: a competing risks analysis. American journal of epidemiology, 173(3), 319-329. https://doi.org/10.1093/aje/kwq368

Baliunas, D. O., Taylor, B. J., Irving, H., Roerecke, M., Patra, J., Mohapatra, S., \& Rehm, J. (2009). Alcohol as a risk factor for type 2 diabetes. Diabetes care, 32(11), 2123-2132. https://doi.org/10.2337/dc09-0227

Bergmann, M. M., Rehm, J., Klipstein-Grobusch, K., Boeing, H., Schütze, M., Drogan, D., ... \& Boutron-Ruault, M. C. (2013). The association of pattern of lifetime alcohol use and cause of death in the European Prospective Investigation into Cancer and Nutrition (EPIC) study. International journal of epidemiology, 42(6), 1772-1790. https://doi.org/10.1093/ije/dyt154

Boffetta, P., \& Hashibe, M. (2006). Alcohol and cancer. The lancet oncology, 7(2), 149-156. https://doi.org/10.1016/S1470-2045(06)70577-0

Di Castelnuovo, A., Costanzo, S., Bagnardi, V., Donati, M. B., Iacoviello, L., \& De Gaetano, G. (2006). Alcohol dosing and total mortality in men and women: an updated meta-analysis of 34 prospective studies. Archives of internal medicine, 166(22), 2437-2445. https://doi.org/10.1001/archinte.166.22.2437

Fillmore, K. M., Kerr, W. C., Stockwell, T., Chikritzhs, T., \& Bostrom, A. (2006). Moderate alcohol use and reduced mortality risk: Systematic error in prospective studies. Addiction Research \& Theory, 14(2), 101-132. https://doi.org/10.1080/16066350500497983

Fuchs, C. S., Stampfer, M. J., Colditz, G. A., Giovannucci, E. L., Manson, J. E., Kawachi, I., ... \& Speizer, F. E. (1995). Alcohol consumption and mortality among women. New England Journal of Medicine, 332(19), 1245-1250. https://doi.org/10.1056/NEJM199505113321901

Klatsky, A. L., \& Udaltsova, N. (2007). Alcohol drinking and total mortality risk. Annals of Epidemiology, 17(5), S63-S67. https://doi.org/10.1016/j.annepidem.2007.01.014

Li, T. K. (2008). Quantifying the risk for alcohol use and alcohol attributable health disorders: Present findings and future research needs. Journal of gastroenterology and hepatology, 23(s1), S2-S8. https://doi.org/10.1111/j.1440-1746.2007.05298.x

Testino, G., Patussi, V., Scafato, E., Ancarani, O., \& Borro, P. (2013). Alcohol, cardiovascular disease and cancer. Alcohol Alcohol, 48(5), 627-628.

McIntosh, J. (2008). Is alcohol consumption good for you? Results from the 2005 Canadian Community Health Survey. Addiction Research \& Theory, 16(6), 553-563. https://doi.org/10.1080/16066350802011631 
-(2014). Alcohol and Type 2 Diabetes: Results from Canadian Cross-Sectional Data. Journal of Diabetes Melitus. 4, 316-323. https://doi.org/10.4236/jdm.2014.44044

-(2014). Obesity and the Demand for Canadian Physician Services. Health, 6, 2624-2631. https://doi.org/10.4236/health.2014.619301

MacMinn W, McIntosh J, Yung C. 2007. How much does obesity matter? Results from the 2001 Canadian Community Health Survey. Advances in Health Economics and Health Services Research: The Economics of Obesity, 17, 333-364. https://doi.org/10.1016/S0731-2199(06)17013-2

O'Keefe, James H, Kevin A. Bybee, and Carl J. Lavie (2007). Alcohol and Cardiovascular Health The Razor-Sharp Double-Edged Sword. Journal of the American College of Cardiology. 50, 1009-1014. https://doi.org/10.1016/j.jacc.2007.04.089

Patra, J., Benjamin, T., Hyacinth, I., Michael, R., Dolly, B., Satya, M., \& Jürgen, R. (2010). RAlcohollconsumption and the risk of morbidity and mortality for different stroke types - a systematic review and meta-analysis. BMC Public Health, 10, 258. https://doi.org/10.1186/1471-2458-10-258

Rehm, J., Patra, J., \& Popova, S. (2006). Alcohol - attributable mortality and potential years of life lost in Canada 2001: implications for prevention and policy. Addiction, 101(3), 373-384. https://doi.org/10.1111/j.1360-0443.2005.01338.x

Rehm, J., Mathers, C., Popova, S., Thavorncharoensap, M., Teerawattananon, Y., \& Patra, J. (2009). Global burden of disease and injury and economic cost attributable to alcohol use and alcohol-use disorders. The Lancet, 373(9682), 2223-2233. https://doi.org/10.1016/S0140-6736(09)60746-7

Roerecke, M., \& Rehm, J. (2010). Irregular heavy drinking occasions and risk of ischemic heart disease: a systematic review and meta-analysis. American Journal of Epidemiology, 171(6), 633-644. https://doi.org/10.1093/aje/kwp451

------- (2012) The cardioprotective association of average alcohol consumption and ischaemic heart disease: a systematic review and meta-analysis. Addiction, 107, 1246-1260. https://doi.org/10.1111/j.1360-0443.2012.03780.x

Room, R., Babor, T., \& Rehm, J. (2005). Alcohol and public health. The lancet, 365(9458), 519-530. https://doi.org/10.1016/S0140-6736(05)70276-2

Statistics Canada (2013). Canadian Community Health Survey, 2011

\footnotetext{
--o13) Alcohol Statistics CANSIM Table

-(2011) National Population Health Survey. (machine readable file) Ottawa Ontario.

Ontario.

(2009). Canadian Community Health Survey, 2007- 2008. (machine readable data file). Ottawa,
}

Stockwell, T. I. M., Butt, P., Beirness, D., Gliksman, L., \& Paradis, C. (2012). The basis for Canada's new low risk drinking guidelines: A relative risk approach to estimating hazardous levels and patterns of alcohol use. Drug and alcohol review, 31(2), 126-134. https://doi.org/10.1111/j.1465-3362.2011.00342.x

\section{Notes}

Note 1. This includes both regular and occasional drinkers since most occasional drinkers also benefit from drinking although not as much as regular drinkers.

\section{Copyrights}

Copyright for this article is retained by the author(s), with first publication rights granted to the journal.

This is an open-access article distributed under the terms and conditions of the Creative Commons Attribution license (http://creativecommons.org/licenses/by/4.0/). 